\title{
Impulse
}

Isabel Laack

\section{Lernziele des religionswissenschaftlichen Studiums: Eine Anregung zur Umsetzung hochschuldidaktischer Erkenntnisse}

Zusammenfassung: Im Zuge der weitreichenden Veränderungen der Universitätslandschaft im letzten Jahrzehnt ist auch die Forderung nach einer Verbesserung der Lehre gestellt worden. In religionswissenschaftlichen Publikationen wurden Fragen der Umsetzung dieser Forderung jedoch bisher erst rudimentär diskutiert. An dieser Stelle setzt vorliegender Artikel an, indem er das zentrale hochschuldidaktische Modell des constructive alignment von Lernzielen, Lehrstrategien und Prüfungen anwendet und Lernziele für das religionswissenschaftliche Studium formuliert. Über welche Fähigkeiten sollen unsere Studenten nach einem erfolgreichen Abschluss des religionswissenschaftlichen Studiums verfügen? Wollen wir ihnen primär religionswissenschaftliches Fachwissen und einen wissenschaftlichen Habitus vermitteln? Oder können und müssen wir auch Lernziele bestimmen, die über das Fachwissen hinausgehen? Mit dieser Fragestellung beschäftigt sich vorliegendes Impulspapier.

\begin{abstract}
The last decade has witnessed many structural changes within German universities. One objective of the new agenda has been to improve the quality of university teaching. In German publications within the field of the academic study of religions, this objective has not yet been much discussed, though. This article wishes to stimulate the debate by introducing fundamental insights from the research on higher education and by applying these to the case of academic teaching about religion. The main emphasis of the paper is to formulate intended learning outcomes within the frame of the 'constructive alignment' model. What is the main objective of teaching the study of religions in higher education? Do we wish to implement solely an academic habitus? Or is it our obligation to define learning outcomes transcending the boundaries of disciplinarily framed factual knowledge? These are the questions this paper is dealing with.
\end{abstract}

DOI 10.1515/zfr-2014-0014

Dr. Isabel Laack: Institut für Religionswissenschaft, Universität Heidelberg, Akademiestraße 4-8, 69117 Heidelberg, Email: isabel.laack@zegk.uni-heidelberg.de 


\section{Einleitung}

Wie wichtig ist das Thema Hochschuldidaktik für die Disziplin Religionswissenschaft? Wieviel Zeit können und möchten an Universitäten angebundene Religionswissenschaftler ${ }^{1}$ in die Hochschullehre investieren? Über welche Fähigkeiten sollen Studenten nach einem erfolgreichen Abschluss des religionswissenschaftlichen Studiums verfügen können? Wollen wir ihnen primär religionswissenschaftliches Fachwissen und einen wissenschaftlichen Habitus vermitteln? Oder können und müssen wir auch Lernziele für das religionswissenschaftliche Studium bestimmen, die über das Fachwissen hinausgehen? Mit dieser Fragestellung beschäftigt sich vorliegendes Impulspapier.

\subsection{Die Ausgangssituation: Die Forderung nach besserer Lehre an den Universitäten}

Meine eigene Erfahrung als Studentin verschiedener geistes- und sozialwissenschaftlicher Fächer an verschiedenen Universitäten Deutschlands ist geprägt von dem Eindruck, dass gute Lehre damals keine besonders große Rolle zu spielen schien. Es gab durchaus Ausnahmen in Form besonders talentierter und engagierter Dozenten. Vorwiegend bestimmt ist meine Erinnerung an die Studienzeit jedoch von Vorlesungen, die vom Blatt abgelesen wurden, und Seminaren mit schlechten Referaten und unstrukturierten, freien und oft ausufernden Diskussionen.

In dem guten Jahrzehnt seit Ende meines Studiums hat sich die deutsche Universitätslandschaft jedoch fundamental verändert, ausgelöst u.a. durch die Bologna-Erklärung europäischer Bildungsminister im Jahr 1999. Diese forderten eine Vereinheitlichung europäischer Studiengänge mit der Absicht, ,international akzeptierte Abschlüsse zu schaffen, die Qualität von Studienangeboten zu verbessern und mehr Beschäftigungsfähigkeit zu vermitteln“ (Bundesministerium für Bildung und Forschung: o.D.). Demzufolge mussten Studiengänge in Deutschland nach dem Bachelor-/Master-System neugestaltet und akkreditiert werden. Dieser Prozess löste gerade in den sogenannten „kleinen“ geisteswissenschaftlichen Fächern Reflexionen über die eigene Identität und die zentralen Inhalte des jeweiligen Fachstudiums aus (vgl. Stausberg 2011, 200). Zusätzlich zur Forderung nach einer strukturellen Vereinheitlichung europäischer Universitätsausbildungen drangen die Bildungsminister auf eine stärkere Ausrichtung des Studiums an

1 Im Folgenden wird das generische Maskulin für Männer und Frauen gleichermaßen verwendet. 
den Erfordernissen des Arbeitsmarkts und eine höhere Berufsfähigkeit (employability) der Graduierten. In diesem Zusammenhang entwickelte sich eine Debatte um die gesellschaftliche Rolle der verschiedenen Disziplinen, die ihre Verwendung öffentlicher Gelder seitdem stärker als je zuvor legitimieren müssen. Gleichzeitig wurde eine Verbesserung der fachbezogenen Ausbildung auf didaktischer Ebene eingefordert (ebd.). Dieser Anspruch wurde bisher v.a. top-down umgesetzt: Universitäten haben zum einen begonnen, hochschuldidaktische Zentren ${ }^{2}$ aufzubauen, zum anderen Instrumente der Lehrevaluation zu entwickeln und in einzelnen Studiengängen einzusetzen. Unklar ist jedoch, ob auch auf Ebene der Fachinstitute und der einzelnen Dozenten eine grundlegende Auseinandersetzung mit der Frage, wie eine bessere Lehre erreicht werden kann, stattfindet. Der Anreiz dazu scheint nicht sehr hoch: Für eine intensive Auseinandersetzung mit hochschuldidaktischen Konzepten bleibt im universitären Alltag selten Zeit; und gute Lehre wird im Gegensatz zur Forschungstätigkeit, zur Anzahl (und hoffentlich auch Qualität) der Publikationen und zur Fähigkeit zum Einwerben von Drittmitteln nach wie vor in unserem Universitätssystem wenig belohnt (vgl. Engler und Stausberg 2011, 132). In Stellenausschreibungen für Universitätslehrer wird dagegen seit einiger Zeit vermehrt der Nachweis von didaktischen Qualifikationen verlangt.

Allein durch externen Druck in Form von Anforderungen an Nachwuchswissenschaftler, in Form von quantitativen Evaluationen der Lehre etablierter Wissenschaftler und in Form von dringlich formulierter Verwaltungsprosa wird die Lehre an den Universitäten jedoch nicht besser - Extrinsische Motivation ist selten ausreichend für nachhaltiges Lernen (vgl. Ramsden 2003, 231-232), erst recht nicht, wenn die Umsetzung des Erlernten gegen viele Widerstände wie Zeitmangel oder Einschränkungen durch die Prüfungsordnung erfolgen müsste. ${ }^{3}$ Schlechte Lehre ist jedoch, wie der Hochschuldidaktiker Paul Ramsden lapidar bemerkt, „a tragic waste of knowledge, experience, youth, time and ability“ sowohl auf Seiten der Studenten als auch der Dozenten $(2003,6)$.

Ich frage mich darüber hinaus, ob eine gute Lehre in der Religionswissenschaft das Potential enthält, die gesellschaftliche Wahrnehmung und Wertschätzung der Disziplin zu erhöhen. Die mangelnde Anerkennung der Religionswissenschaft als förderungswürdiges Forschungsfeld und von Religionswissenschaftlern als Ansprechpartnern in gesellschaftlich relevanten Fragen macht viele Fachvertreter sehr unzufrieden, wie in den immer wieder aufflammenden Diskussionen in

2 Vgl. das Hochschuldidaktikzentrum der Universitäten Baden-Württembergs, http://www.hdzbawue.de.

3 Vgl. für eine umfassende Diskussion der Evaluation und Verbesserung von Lehre auf Institutsund Universitätsebene Ramsden (2003, 207-254). 
der religionswissenschaftlichen Emailliste Yggdrasill deutlich wird. ${ }^{4}$ Ich halte es für durchaus vorstellbar, dass graduierte Religionswissenschaftler, die systematisch, kreativ, informiert, reflektiert und ethisch verantwortungsvoll in ihren Berufen agieren, nicht nur das Wissen um die Existenz der Disziplin verbreiten, sondern auch deren Wahrnehmung als kompetenter Ansprechpartner verbessern. Um das Ziel gut ausgebildeter Religionswissenschaftler zu erreichen, ist eine gute Lehre unabdingbar. Diese kann zum einen die Beschäftigung mit aktuellen, gesellschaftspolitisch relevanten Themen im Studium umfassen. Darüber hinaus sollte meiner Meinung nach jedoch auch bei nicht unmittelbar anwendbaren Gegenständen der Religionswissenschaft eine Didaktik eingesetzt werden, die unsere Studenten zu nachhaltigem Lernen ermutigt und es den Graduierten ermöglicht, fachtypische Denkstrategien, Methodenansätze und Problemlösungsstrategien in ihrem beruflichen Alltag einzusetzen. Bei dieser Art von „Anwendung“ durch die Lehre geht es nicht um die Reduktion der Disziplin auf eine Anwendungswissenschaft, sondern um das didaktische Vermitteln der Relevanz der Religionswissenschaft. Auch und gerade historische Themen und die Beschäftigung mit weit von uns entfernten Kulturen und Religionen können relevant für unser Lernen sein - relevant für das Nachdenken über grundlegende Menschheitsfragen, für ein besseres Verstehen der Motivationen und Formen menschlichen Handelns oder der Entwicklungslinien ganzer Kulturen und Gesellschaften. Dieses Nachdenken durch eine gute Lehre zu fördern und die Graduierten mit diesem Potential in die Welt außerhalb der Universität zu entlassen, könnte ein wichtiges Ziel und eine intrinsische Motivation zur Verbesserung der Lehre in der universitären Religionswissenschaft darstellen.

Welche Schritte in diese Richtung wurden schon unternommen? Im Folgenden gebe ich zunächst einmal einen Überblick über den gegenwärtigen Diskussionsstand in Form von Publikationen zur Umsetzung didaktischer Fragen in der Religionswissenschaft.

\subsection{Der Publikationsstand: Religionswissenschaftliche Didaktik $^{5}$}

Die Hochschuldidaktik als Teilbereich der wissenschaftlichen Disziplin der Pädagogik hat in den letzten Jahrzehnten durch ihre Forschung viele wertvolle Er-

\footnotetext{
4 Vgl. auch die Tagung der DVRW 2011 in Heidelberg zum Thema: „Religionswissenschaft im Aufwind. Eine Profilbestimmung angesichts steigender gesellschaftlicher Relevanz“.

5 Ich danke meiner wissenschaftlichen Hilfskraft Adrian Heinrich für seine Hilfe bei der Literaturrecherche und die wertvollen inhaltlichen Anmerkungen.
} 
kenntnisse über menschliches Lernen im Allgemeinen und an Universitäten im Besonderen gewonnen. Eine Rezeption und Umsetzung dieser Erkenntnisse in anderen Disziplinen lässt jedoch noch zu wünschen übrig. Zwar ist die Beschäftigung mit Schlüsselkompetenzen seit dem Bologna-Prozess angestiegen (vgl. Nünning 2008). Konkrete Umsetzungen didaktischer Erkenntnisse findet man für geistes- und kulturwissenschaftliche Fächer erst vereinzelt. ${ }^{6}$

Ähnlich dürftig sieht die Lage der Publikationen $\mathrm{zu}$ diesem Thema in der Religionswissenschaft aus. Im Mittelpunkt der Überlegungen im Zuge der Gestaltung neuer Studiengänge nach dem Bachelor-/Master-Modell stand die Festlegung von zentralem Fachwissen und erforderlichen Methoden und Sprachen; darüber hinaus die Verankerung der Förderung von Schlüsselkompetenzen im Studium, um der Forderung nach höherer employability der Graduierten nachzukommen (vgl. Deutsche Vereinigung für Religionswissenschaft 2011). Fragen über den Zweck des religionswissenschaftlichen Studiums und seine pädagogische Ausrichtung wurden in einem Symposium der Tagung der International Association for the History of Religion 2010 in Toronto angesprochen. Ergebnisse dieser von Michael Stausberg organisierten Meta-Reflektion über Prozesse der Umstrukturierung in der Religionswissenschaft seit dem Bologna-Prozess und weltweit ähnlichen Entwicklungen erschienen 2011 in einem Sonderheft der Zeitschrift Religion 42/2 (vgl. Stausberg 2011; Engler und Stausberg 2011, 127).

Ein relativ junges Thema in der Religionswissenschaft (zumindest in der deutschsprachigen) ist die Diskussion der Funktion der Disziplin in der Ausbildung von schulischen Religionslehrern. Diese insbesondere von Wanda Alberts initiierte Debatte ${ }^{7}$ diskutiert auf sehr grundlegender Ebene, wie schulischer Religionsunterricht aussehen kann, der von den Prämissen einer kulturwissenschaftlichen Religionswissenschaft geprägt ist (im Gegensatz zu separativen, theologischen und religionspädagogischen oder integrativen interreligiösen Ansätzen, vgl. Alberts 2008a). Obwohl sich manche Fragen mit der Hochschuldidaktik überschneiden - z.B. wie und $\mathrm{zu}$ welchem Zweck religionswissenschaftliche Theorien vermittelt oder Religionen kulturwissenschaftlich unterrichtet werden können -, werden konkretere Themen didaktischer Umsetzung an den Universitäten bisher nur am Rande gestreift (z. B. in Frank und Bochinger 2008, 212-214).

Die Frage nach der gesellschaftlichen Rolle der Religionswissenschaft und damit verbunden auch die nach den Lernzielen des religionswissenschaftlichen

6 Ein vorbildliches Beispiel dafür ist der von Irina Bauder-Begerow und Stefanie Schäfer herausgegebene Sammelband (2011a). Vgl. auch die inspirierende Modularbeit im Weiterbildungsstudiengang Hochschuldidaktik der Universität Bern von Petra Bleisch und Dirk Johannsen (o. D.).

7 Vgl. Alberts’ „Editorial.“ In Michael Stausberg, Hg., Sonderheft The Challenge of Religious Education for the History of Religions. Numen 55: 121-122. 
Studiums wird in zwei aus der Perspektive der Deutschen Vereinigung für Religionswissenschaft (DVRW) verfassten Aufsätzen behandelt. So präsentierte Christoph Bochinger, derzeitiger Vorsitzende der DVRW, in einem Vortrag auf dem Kongress des Wissenschaftsrates 2010 zum Thema „Vielfalt der Religionen - Theologie im Plural“ Thesen über den Nutzen des Faches für die Gesellschaft (Bochinger 2010). Ein Jahr später veröffentlichte die DVRW ihre „Empfehlungen für die Konzeption religionswissenschaftlicher Studiengänge an deutschen Universitäten“ (Deutsche Vereinigung für Religionswissenschaft 2011). Darin werden neben konkreten Fragen der modularen Strukturierung von Studiengängen grundlegende fachbezogene Inhalte aufgelistet, die jeder religionswissenschaftliche Studiengang lehren sollte (z.B. Religions- und Kulturtheorien, Begriffe der Religionswissenschaft, Religionsgeschichte) (Deutsche Vereinigung für Religionswissenschaft 2011, 5-6). Zusätzlich werden kurz allgemeine Lernziele jedes „kultur-, geistes- oder sozialwissenschaftlichen Studiums“ erwähnt: die Kompetenz zu lebenslangem Lernen, das Ziel ,junge Menschen zu selbständigem Denken und zu verantwortungsbewusstem Handeln zu befähigen“ sowie die Vermittlung ,allgemeine[r] Schlüsselkompetenzen wie die Fähigkeit zu[r] schnellen Informationsgewinnung, zur kritischen Würdigung und reflektierten Auswertung der gewonnenen Informationen und zu deren nutzbringender Anwendung auf konkrete Problemstellungen“ (Deutsche Vereinigung für Religionswissenschaft 2011, 5). Charakteristisch für den derzeitigen Stand der Auseinandersetzung mit didaktischen Fragen in der deutschen Religionswissenschaft ist, was nicht in dieser Empfehlung thematisiert wird: die Frage, wie die erwähnten allgemeinen Lernziele eines kultur-, geistesoder sozialwissenschaftlichen Studiums oder die fachbezogenen Lernziele der Religionswissenschaft didaktisch vermittelt werden können.

Zusammenfassend lässt sich zum Publikationsstand der Auseinandersetzung mit Hochschuldidaktik in der Religionswissenschaft Folgendes festhalten: Der Bologna-Prozess stimulierte Bestimmungen fachbezogener Kernthemen und förderte den formalen Einbezug der Vermittlung von Schlüsselkompetenzen in das Fachstudium. Neben einer ersten Metareflexion über die strukturellen Veränderungsprozesse an den Universitäten wird auch die gesellschaftliche Rolle und Funktion der Religionswissenschaft vermehrt diskutiert. Darüber hinaus ist eine Forschungsrichtung entstanden, die sich mit der Entwicklung kulturwissenschaftlich-religionswissenschaftlicher Ansätze für den schulischen Religionsunterricht beschäftigt. Es bleibt die Aufgabe, diese verschiedenen Diskussionsstränge miteinander zu verknüpfen und zu erarbeiten, wie Erkenntnisse aus der Hochschuldidaktik konkret in die alltägliche Praxis der universitären Lehre umgesetzt werden können. 


\subsection{Das Vorhaben: Entwicklung von Lernzielen für das religionswissenschaftliche Studium}

An dieser Stelle will vorliegendes Impulspapier ansetzen. Als erster Beitrag zur Debatte wird im Folgenden diskutiert, welche allgemeinen, über Fachwissen hinaus gehenden Lernziele für das religionswissenschaftliche Studium bestimmt werden können. Über welche Fähigkeiten sollen Studenten nach einem erfolgreichen Abschluss des religionswissenschaftlichen Studiums verfügen können? Mit dieser Fragestellung wird eine Auseinandersetzung mit theoretischen Grundlagen der Hochschuldidaktik in Form ihrer Anwendung auf die religionswissenschaftliche Lehre angestrebt.

$\mathrm{Zu}$ diesem Zweck stelle ich zunächst (2) das allgemein anerkannte hochschuldidaktische Konzept des constructive alignment von Lernzielen, Lehrstrategien und Prüfungen vor, das auf die Bestimmung von Lernzielen im folgenden Abschnitt (3) vorbereitet.

Diese hochschuldidaktischen Grundlagen werden dann (4) auf die Religionswissenschaft angewendet. Zuerst (4.1) unterscheide ich fachbezogene von allgemeinen Lernzielen und streife dabei die Frage über den Zweck und den gesellschaftlichen Nutzen eines religionswissenschaftlichen Studiums. In der Debatte um die zunehmende Vermarktung universitärer Bildung hilft ein Exkurs (4.2) zu den primär die Persönlichkeitsbildung betreffenden Lernzielen der General Education an der Harvard University. Schließlich (4.3) formuliere ich konkrete Lernziele, unterteilt in (4.3.1) allgemeine Lernziele eines kultur- und geisteswissenschaftlichen Studiums und (4.3.2) spezifische Lernziele der Religionswissenschaft. In der Abschlussdiskussion (5) fasse ich die Ergebnisse des Artikels zusammen und verweise auf die Bedeutung der Hochschuldidaktik für die Verbesserung unserer Studiengänge.

\section{Hochschuldidaktische Grundlagen}

Als Einstieg in die hochschuldidaktische Perspektive ist es hilfreich, sehr grundsätzlich zu reflektieren, was dort unter Lernen verstanden wird. Paul Ramsden weist in seinem hochschuldidaktischen Klassiker Learning to Teach in Higher Education (Ramsden 2003) ${ }^{8}$ darauf hin, dass Lernen weit über die bloße Aneignung von Fakten hinausgeht. Lernen hat erst dann stattgefunden, wenn sich das

8 Im vorliegenden Artikel wird die zweite Auflage von 2003 verwendet; inzwischen ist eine neue Auflage erschienen. 
Verstehen der Welt um uns herum und unsere Konzeptualisierung dieser Welt verändert hat (Ramsden 2003, 6.41.79). Eine konsequente Umsetzung dieser Perspektive auf das Lernen setzt vorrangig an einer Veränderung der Einstellung der Lehrer an. Dabei geht es Ramsden nicht um blinden Aktionismus oder den willkürlichen Einsatz neumodischer, unterhaltsamer und extravaganter Methoden. Zwar ist es wichtig, Lehrmethoden und -strategien einzusetzen, die Alternativen zur klassischen Vorlesung oder freien Diskussion darstellen. Jedoch führt der Einsatz dieser Methoden allein noch nicht zu besserer Lehre, nur ihre flexible und gezielte Anwendung (vgl. Ramsden 2003, 81.109.120.244.253). Der theoretische Hintergrund dieser Perspektive auf Lernen ist der didaktische Konstruktivismus und das student learning research paradigm (Biggs 2012, 119; Biggs und Tang 2009, 20-21). Demnach sind Lernende keine passiven Rezipienten von Lernstoff, sondern konstruieren aktiv ihr angeeignetes Wissen (Biggs 2012, 125). Im landläufigen Unterricht werden dagegen „learning pathologies“ gezüchtet, wie es Biggs (2012, 118) prägnant auf den Punkt bringt, denn in ihm werden sogenannte surface approaches to learning gefördert. Die Unterscheidung zwischen deep learning bzw. high quality learning und surface learning ist inzwischen allgemein anerkannter Standard in der Didaktik. ${ }^{9}$ Surface Learning bleibt an der Oberfläche und bezieht sich meist auf die unreflektierte Übernahme von Faktenwissen und eindimensionaler Kenntnisse von Methoden und Theorien. Der Inhalt dieses oberflächlich Gelernten wird schnell wieder vergessen und hat keinen langfristigen Nutzen für den Lernenden (vgl. Ramsden 2003, 43). Nach einem Prozess des Deep Learning dagegen hat sich der Lernende mit dem Lernstoff auseinandergesetzt und hat sich sein Verstehen der Welt verändert. Das Ergebnis von High Quality Learning ist umfassendes, miteinander verknüpftes und komplex strukturiertes Wissen, das fruchtbar eingesetzt und auf andere Kontexte übertragen werden kann und das dem Lernenden langfristig erhalten bleibt (Kirby und Lawson 2012b, 2-4). Effektives Tiefen-Lernen wird durch komplexe Faktoren in vielen verschiedenen Bereichen des Lernens und seiner Umgebung gefördert (oder verhindert) (vgl. Ramsden 2003, 74-98). Neben der Berücksichtigung der vom Dozenten geschaffenen Lehrumgebung oder dem gezielten Einsatz von verschiedenen Lehrmethoden stellt das sogenannte constructive alignment nach John Biggs (vgl. Biggs 2012) ${ }^{10}$ eine wesentliche Säule dieser Strategien dar. Es ist ein

\footnotetext{
9 Vgl. die Sammlung aktueller Forschungsergebnisse zu den Aspekten der Disposition der Lernenden sowie Lernbedingungen, -anforderungen und -strategien im „high quality learning“ in Kirby und Lawson (2012a). Vgl. zur Begriffs- und Forschungsgeschichte des „deep learning“ Lawson und Askell-Williams (2012, 140-142) und Biggs (2012, 120).

10 Vgl. zur Entstehung des Modells Biggs und Tang $(2009,50)$.
} 
inzwischen weiträumig in der Praxis erprobtes Modell zur Förderung des High Quality Learning (vgl. Ramsden 2003, 66.122; Kirby und Lawson 2012, 4-5).

Dem Modell des Constructive Alignment folgend müssen im Rahmen des konstruktiven Lernparadigmas drei Stadien des Designs von Kursen aufeinander abgestimmt werden, um tiefenorientiertes Lernen zu fördern: die Bestimmung der Lernziele, die Auswahl der Lehrstrategien und die Abfrage des Lernerfolgs in Prüfungen. Die Prinzipien, denen diese Abstimmung folgt, sind grundsätzlich sehr einfach (Biggs 2012, 124):

„1. Define what students are supposed to do as a result of having been taught a topic.

2. Engage them in learning activities that are most likely to help them achieve the outcome defined in (1).

3. Assess to see how well they have achieved the desired outcomes. "11

Diese Prinzipien können sowohl für die Gestaltung einzelner Lehrveranstaltungen eingesetzt werden als auch für die Evaluation von Lehre und für die Erhöhung des Lehrstandards auf der Ebene von Institutionen (Ramsden 2003, 119120).

Das Constructive Alignment verschiebt die Perspektive von einer bloßen Aufzählung von Lerninhalten hin zu einer Orientierung am Lernenden (Biggs 2012, 123.126). Als Erstes überlegen Dozenten, was die Absolventen eines Kurses hinterher gelernt und welches Niveau sie dabei erreicht haben sollen. Als Nächstes werden die Lehrstrategien und -methoden auf die Lernziele abgestimmt. Am effektivsten ist es, die Studenten schon im Kurs üben zu lassen, was sie hinterher können sollen (Biggs 2012, 125.128-129). Dafür können gezielt Lehrmethoden eingesetzt werden, die diese Tätigkeiten fördern. ${ }^{12}$ Nach Bestimmung der Lernziele und Auswahl der Lehrstrategien müssen schließlich die Prüfungsformen und -inhalte auf die Lernziele abgestimmt werden. Ramsden weist darauf hin, dass Prüfungen nicht nur das Ergebnis von Lernen widerspiegeln, sondern als zentraler Teil des Lernprozesses zu verstehen sind, weil sie das Lernen steuern (Ramsden 2003, 176-184.205-206). Vor dem Hintergrund unserer an nachweisbarer Leistung orientierten Gesellschaft passen Studenten natürlicherweise ihre Lernstrategien dem an, was Lehrer von ihnen erwarten und am Ende eines Kurses abfragen und bewerten: „From our students’ point of view, assessment always defines the actual curriculum“ (Ramsden 2003, 182). Die schönste Formulierung von Lernzielen und der beste Einsatz von Lehrmethoden führen zu nichts, wenn die Prüfung etwas

11 Vgl. die etwas ausführlichere Variante dieser Prinzipien in Biggs und Tang (2009, 54-55).

12 Für eine Sammlung erprobter Methoden vgl. Macke, Hanke und Viehmann 2008, 146-232. 
völlig anderes, nämlich Oberflächenwissen, von den Studenten abfragt (vgl. Ramsden 2003, 122). Als letzter Punkt, der nicht formal zum Constructive Alignment gehört, sollte erwähnt werden, wie wichtig für langfristige Lernerfolge das Feedback auf die Leistungen von Studenten ist. Nur durch ein angemessen ausführliches, konstruktives und zeitnah gegebenes Feedback kann sich ein Lernender weiterentwickeln (vgl. Ramsden 2003, 74-75).

Die Problematik des Modells liegt natürlich in seiner Umsetzung in den Lehralltag. Als Hochschullehrer sind wir zum einen an Vorgaben der Prüfungsordnungen gebunden, die uns mehr oder weniger Spielraum für die Gestaltung der Lehrinhalte und Prüfungsformen lässt. In den Ansätzen der Bologna-Reformen lässt sich zudem ein gewisser Widerspruch sehen: Auf der einen Seite wird eine bessere Lehre gefordert und erwartet, dass die Graduierten besser befähigt werden, ihr neues Wissen und ihre erworbenen Kompetenzen nach ihrem Studium in ihre jeweiligen Berufsfelder transferieren zu können. Auf der anderen Seite geht die Tendenz dahin, wieder mehr oberflächliches Faktenwissen abzufragen, um für die Benotung eine höhere Objektivität, Vergleichbarkeit und juristische Absicherung zu erreichen. Zusätzlich zu diesen systemischen Einschränkungen der eigenen didaktischen Handlungsfähigkeit sehen sich Hochschullehrer auch im praktischen Alltag mit dem allmächtigen Zeitproblem konfrontiert. High Quality Learning und die Begleitung der Lehrenden in diesem Prozess gelingt am besten bei einem kleinen Betreuungsschlüssel. Diese Widerstände sollten uns jedoch nicht davon abhalten, uns mit hochschuldidaktischen Erkenntnissen und den Möglichkeiten (und Unmöglichkeiten?) ihrer Umsetzung in der Religionswissenschaft auseinanderzusetzen.

\section{Die Bestimmung von Lernzielen}

Nach dieser Einführung in das hochschuldidaktische Modell des Constructive Alignment soll eines seiner Aspekte im Detail beleuchtet werden: die Formulierung von Lernzielen.

Die Formulierung von Lernzielen hat zweierlei Auswirkungen: Zum einen hilft es dem Dozenten, die Perspektive der Lernenden einzunehmen, die Lernziele eines Kurses (oder eines Studiengangs) aus diesem Blickwinkel heraus zu reflektieren und im Sinne des Constructive Alignment Lehrmethoden und Prüfungen (im Rahmen der prüfungsrechtlichen Möglichkeiten) darauf abzustimmen. Zum anderen hilft es Studenten, sich im Lernstoff zu orientieren und den größeren Rahmen im Blick zu behalten, anstatt sich in Details zu verlieren. Darüber hinaus können sie die Erwartungen, die an ihr Lernen gestellt werden, besser einschätzen. Aus diesem zweiten Grund ist es fundamental wichtig, Lernziele den Studen- 
ten so direkt und klar formuliert wie möglich mitzuteilen. Der Lernerfolg ist dadurch erwiesenermaßen höher (vgl. Ramsden 2003, 74-87.98).

\subsection{Grundlagen zur Formulierung von Lernzielen}

Als Erleichterung für den Prozess der Formulierung von Lernzielen ist es hilfreich, sich folgende Prinzipien bewusst $\mathrm{zu}$ halten (Ramsden 2003, 126): 1) Lehre zielt darauf ab, Veränderungen im Wissen, Denken und Verhalten der Studenten hervorzurufen. 2) Es fördert den Lernerfolg, Studenten zu Beginn eines Kurses so präzise und verständlich wie möglich die Lernziele zu erklären. 3) Ob tatsächlich Lernen stattfindet, hängt davon ab, was die Lernenden tun und nicht, was der Lehrer tut.

Klassische Kommentare in Vorlesungsverzeichnissen dagegen lassen ein Bewusstsein dieser Prinzipien i.d.R. vermissen. Anstelle von Lernzielen werden meist nur zentrale Punkte der Lerninhalte aufgezählt und die im Kurs eingesetzten Arbeitsformen erwähnt (vgl. Ramsden 2003, 24). Um aus den alten Formulierungen von Kommentaren oder Syllabi auszubrechen, schlagen Gerd Macke, Ulrike Hanke und Pauline Viehmann folgendes Vorgehen vor (Macke, Hanke und Viehmann 2008, 76):

1. Zunächst wird die Lernzielart benannt; in den Kulturwissenschaften an Universitäten sind es i.d.R. kognitive Lernziele (im Gegensatz zu affektiven oder psychosomatischen).

2. Anschließend werden die Lernziele als Handlungsziele formuliert: Lehrinhalte werden mit einer Tätigkeit verknüpft; z.B.: Studenten können die Religionstheorie Eliades in eigenen Worten erklären.

3. Die Lernziele (pro Seminar etwa fünf bis sechs) werden nach Schwierigkeitsgrad und Komplexität geordnet.

4. Schließlich wird eine Prüfungsform ausgewählt, die erfassen kann, ob die Lernziele erreicht worden sind.

Zwei dieser Arbeitsschritte bespreche ich im Einzelnen: die Formulierung von Lernzielen als Handlungsziele (Punkt 2) und die Ordnung von Lernzielen nach ihrem Schwierigkeitsgrad (Punkt 3).

\subsection{Lernziele als Handlungsziele}

In der didaktischen Forschung ist es umstritten, inwieweit Lernziele als Tätigkeiten aufgefasst werden sollen. So wurden die frühen, stark behavioristischen 
Modelle des Lernens als $\mathrm{zu}$ einseitig und $\mathrm{zu}$ eng kritisiert (vgl. Ramsden 2003, 125.127-128; Biggs 2012, 124). Natürlich wollen Geisteswissenschaften oft, dass Studenten eine Theorie o. ä. intellektuell verstehen; und verstehen gilt in engen behavioristischen Definitionen nicht als Tätigkeit. Eine pragmatische Antwort auf diese Debatten ist, es sich bei der Formulierung der Lernziele nicht zu einfach machen. Werden die klassischen Aufzählungen von Elementen des Lernstoffs nur durch das Verb „verstehen“ ergänzt, bleibt unbestimmt, bis zu welchem Komplexitätsgrad verstehen stattfinden soll, welche Lehrstrategien eingesetzt werden, um dieses Verstehen zu ermöglichen und wie es abschließend abgefragt werden kann (vgl. Ramsden 2003, 126; Biggs und Tang 2009, 74-76). Aus diesem Grund ist es ratsam, eine Tätigkeit einzubeziehen: Die Studenten sollen die Theorie nicht nur verstehen, sondern z.B. auch imstande sein, dieses Verständnis verbal auszudrücken, also die Theorie zu erklären.

Weiterhin sollten Lernziele nicht zu unkonkret benannt werden, z.B. „Die Studenten sollen ein Bewusstsein für die Historizität von wissenschaftlichen Theorien entwickelt haben“. Stattdessen müssen - zumindest auf der Ebene einzelner Seminare - präzisere Aussagen getroffen werden, damit die Lernziele verständlich, umsetzbar und prüfbar werden; z.B. „Die Studenten haben ein Bewusstsein für den historischen Kontext von Eliades Religionstheorie entwickelt und können erklären, inwiefern dieser Kontext Auswirkungen auf seine Theorie hat“.

\subsection{Komplexitätsgrade von Lernzielen}

In Lernzielen wird nicht nur definiert, was gelernt werden soll, sondern auch bis zu welchem Niveau (vgl. Biggs 2012, 126). In der hochschuldidaktischen Literatur kursieren verschiedene Modelle zur Erschließung von Komplexitätsgraden intellektueller Vorgänge und Handlungen. Eines der berühmtesten ist die Taxonomie, die von Benjamin Bloom und einer Arbeitsgruppe von Lehrern schon in den 1950er Jahren entworfen wurde (vgl. Bloom 1956-1964). ${ }^{13}$ Diese Taxonomie kann in einer Reihe von Verben zusammengefasst werden: wissen, verstehen, anwenden, analysieren, synthetisieren und bewerten. ${ }^{14}$ Diese intellektuellen Vorgänge

13 Vgl. für einen Überblick über weitere Modelle Lawson und Askell-Williams (2012, 143-150). Diese z.T. jüngeren Modelle sind zwar besser in empirischen Studien verankert (vgl. Bereiter und Scardamalia 2012, 173) und bilden mehr Komplexität ab als die klassischen Modelle von Bloom et al. und Biggs; letztere sind aber für die pragmatische Umsetzung in die Lehre nach wie vor hilfreich.

14 Vgl. für eine ausführlichere Erklärung, welche kognitiven Fähigkeiten mit diesen Verben verbunden werden, Macke, Hanke und Viehmann $(2008,79)$. 
bauen aufeinander auf; verstehen ist nicht möglich ohne wissen, anwenden nicht ohne verstehen, und bewerten nicht ohne die vorausgehenden Denkprozesse. Deshalb müssen in Prüfungen auch nicht alle Schritte einzeln abgeprüft werden, sondern es kann an den höherstehenden im Schema angesetzt werden. Die Bloom'sche Taxonomie kann zusätzlich zur Formulierung von Lernzielen auch zur Bewertung von Leistungen eingesetzt werden; je höher die Stufe ist, die ein Student erreicht hat, desto besser ist seine Bewertung.

Ein ähnliches Modell ist die Structure of Observed Learning Outcomes (SOLO)Taxonomie nach John Biggs, die ebenfalls intellektuelle Fähigkeiten im Umgang mit Lernstoff nach ihrer Komplexität ordnet, in ihrer Konzeption jedoch von der Idee des Deep Learning geprägt ist und auf der Grundlage von Forschungsergebnissen mit Lernenden entwickelt wurde (Biggs 2012, 122.127; Biggs und Tang 2009, 27.77-81). Biggs und Tang ordnen den einzelnen Stufen eine ganze Reihe von Verben $\mathrm{zu}$, die als Orientierung für die Formulierung von Lernzielen dienen können.

1. Die niedrigste Stufe benennt unistrukturelles, eindimensionales Lernen; d.h. einzelne Aspekte des Lernstoffs werden erinnert und können wiedergegeben werden (vergleichbar mit Blooms „wissen“). Mit der ersten Stufe verbundene Verben sind: erinnern, identifizieren, erkennen, zählen, definieren, zeichnen, finden, benennen, zitieren, ordnen, erzählen, schreiben oder imitieren.

2. Auf der zweiten, multistrukturellen Stufe werden mehrere Aspekte erinnert, die aber noch unverbunden miteinander stehen (vergleichbar mit Blooms „wissen“ in Bezug auf eine größere Menge von Lernstoff, evtl. auch schon Ansätze des „verstehen“); passende Verben sind: klassifizieren, beschreiben, auflisten, diskutieren, illustrieren oder auswählen.

3. Auf der dritten, relationalen Stufe können Lernende mehrere Aspekte miteinander verbinden und in ein Ganzes integrieren (Blooms „,verstehen“, „anwenden“, „analysieren“ und „synthetisieren“). Diese Fähigkeit wird eingesetzt bei Tätigkeiten wie: anwenden, integrieren, analysieren, erklären, vorhersagen, zusammenfassen, argumentieren, übertragen, einen Plan machen, charakterisieren, vergleichen, kontrastieren, differenzieren, organisieren, debattieren, konstruieren, überarbeiten, untersuchen, übersetzen, paraphrasieren, ein Problem lösen.

4. Haben Lernende die höchste Stufe des „extended abstract“ erreicht, sind sie imstande, das Erlernte zu generalisieren und eigenständig in abstraktere Denkmodelle zu überführen; sie können theoretisieren, Hypothesen aufstellen, generalisieren, reflektieren, generieren, erschaffen, erfinden, Probleme auf der Grundlage von Prinzipien lösen und eigene Thesen aufstellen (Blooms „bewerten“ ist ein Teil dieser Fähigkeit). (Biggs und Tang 2009, 81) 
Nach Einschätzung von Biggs findet Deep Learning ab der dritten Stufe statt, auf der Lernende Inhalte miteinander verbinden und sie anwenden können. Ähnlich wie die Bloom'sche Taxonomie kann SOLO als Maßstab für die Bewertung von Prüfungsleistungen eingesetzt werden, indem Bewertungskategorien mit der Taxonomie parallelisiert und die Leistungen daraufhin analysiert werden, welche Stufe die Studenten erreicht haben (vgl. Biggs und Tang 2009, 58-59).

\section{Lernziele des religionswissenschaftlichen Studiums}

Nach der Vorstellung von Prinzipien und Hilfestellungen zur Formulierung von Lernzielen möchte ich nun diese hochschuldidaktischen Grundlagen auf das Fach der Religionswissenschaft anwenden. Dafür skizziere ich zunächst einige Eckpunkte der Debatte um den gesellschaftlichen Nutzen des religionswissenschaftlichen Studiums und ergänze sie durch einen Exkurs zu den Lernzielen der Harvard General Education. Anschließend entwickele ich allgemeine Lernziele sowohl der Geistes- und Kulturwissenschaften insgesamt als auch der Religionswissenschaft im Besonderen.

\subsection{Der gesellschaftliche Nutzen des religionswissenschaftlichen Studiums}

Die Bestimmung von Lernzielen eines Studiengangs wird davon beeinflusst, welchen Sinn und Zweck wir ihm grundsätzlich zusprechen. In jedem Universitätsstudium werden unterschiedliche Typen von Lerninhalten vermittelt: fachbezogene Inhalte, allgemeine Lernziele und berufsbezogene Kompetenzen. In den präBologna-Studiengängen wurden meist primär fachbezogene Inhalte gelehrt, und sie stehen auch immer noch im Zentrum religionswissenschaftlicher Ausbildung. ${ }^{15}$ Seit Bologna müssen Institute jedoch auch legitimieren, für welche Berufsfelder ihr Studiengang ausbildet. Der vermutlich einzige direkt fachbezogene Beruf der Religionswissenschaft ist der Akademiker; und ein Religionswissenschafts-Bachelor muss für das darauf aufbauende Master-Studium und eine even-

15 Auch die erwähnten Empfehlungen der DVRW zur Gestaltung von religionswissenschaftlichen Studiengängen widmen sich hauptsächlich fachbezogenen Inhalten (Deutsche Vereinigung für Religionswissenschaft 2011). 
tuelle akademische Laufbahn vorbereiten. Daneben muss er jedoch auch den Anforderungen an eine höhere Employability der Graduierten gerecht werden und berufsbezogene Fähigkeiten im Studium unterrichten. Dementsprechend führen religionswissenschaftliche Institute neuerdings auf ihren Homepages und Werbematerialien für den Studiengang mögliche Berufsfelder für Religionswissenschaftler an (z. B. Journalismus, Politikberatung, Integrationsarbeit von Immigranten, etc., vgl. Deutsche Vereinigung für Religionswissenschaft 2011, 7).

Die Vermittlung berufsbezogener Kompetenzen findet jedoch bisher oft auf einer oberflächlichen Ebene statt, z. B. durch ein Praktikum als Pflichtmodul (vgl. Berner 2011, 149) oder das Angebot spezieller Kurse für Schlüsselkompetenzen wie Zeitmanagement, Präsentieren in Vorträgen oder der Umgang mit modernen Wissenstechnologien (wie im dualen Modell in Heidelberg, vgl. Chur 2011). ${ }^{16}$ Mir ist dagegen nicht bekannt, ob religionswissenschaftliche Institute schon den Versuch unternommen haben, ausführlich zu reflektieren, welche Schlüsselkompetenzen in den von ihnen genannten Berufsfeldern erwartet werden und wie diese im normalen Unterricht gelehrt werden können. ${ }^{17}$

In Abgrenzung zur Forderung der stärkeren Berufsausbildung im Studium gibt es Stimmen, die diesen Trend zur Kommerzialisierung, Ökonomisierung und Vermarktung des Studiums kritisieren (vgl. Engler und Stausberg 2011, 138; Alles 2011). Interessanterweise scheint die Employability auch kein starkes Motiv bei Studenten für die Wahl des Studienfachs Religionswissenschaft zu sein; das ist zumindest eines der Ergebnisse der explorativen Studie mit Studenten aus Göttingen und Hannover, die eine Forschergruppe um Steffen Führding durchgeführt hat (Führding et al. 2009). Stattdessen äußerten einige der befragten Studenten sogar ihre Motivation, Religionswissenschaft zu studieren, obwohl sie vermuten, als Graduierte einer prekären Situation auf dem Arbeitsmarkt ausgesetzt zu sein: „Religionswissenschaft wird insgesamt nicht wegen, sondern trotz der beruflichen Perspektiven studiert" (Führding et al. 2009, 144).

Zusätzlich zu fach- und berufsbezogenen Arten von Lernzielen lässt sich ein gesellschaftlicher Nutzen des religionswissenschaftlichen Studiums benennen: die Bildung ihrer Studenten zu mündigen Bürgern unserer globalisierten Welt (vgl. Engler und Stausberg 2011, 137). Um den Herausforderungen einer sich schnell verändernden, multikulturellen und globalisierten Welt gewachsen zu sein, brau-

16 Eine weitere Strategie ist es, den Erwerb berufsbezogener Kompetenzen vollständig aus dem Studium auszulagern und zu argumentieren, dass jener Aufgabe von Ausbildungen ist, die auf das Studium folgen, wie z. B. dem Volontariat.

17 Z.B. wäre es möglich (und wird auch schon in einigen Kursen von Dozenten umgesetzt), in Kursen nicht nur wissenschaftliches Schreiben, sondern gezielt auch journalistische Schreibtechniken und -stile einzuüben. 
chen junge Generationen weitere Kompetenzen als nur technische Fähigkeiten oder fachbezogenes Wissen. So weisen Michael Stausberg und Steven Engler denn auch auf die wichtige Rolle der Universitäten zur Vermittlung zentraler Werte einer post-säkularen Weltgesellschaft hin (ebd.).

Über diesen zwar nicht unmittelbar ökonomischen, aber gesellschaftlichen Zweck des religionswissenschaftlichen Studiums hinaus gibt es auch scheinbar „nutzfreie“ Gründe, diesen Studiengang anzubieten. So ruht das Humboldt'sche Bildungsideal nicht nur auf einer engen Verzahnung von Forschung und Lehre in einer Lernumgebung des direkten Kontakts zwischen Professoren und Studenten. Darüber hinaus verweist es auf die Ideen der Persönlichkeitsbildung, der Bereicherung des Lebens durch Bildung und des Lernens um des Lernens willen (vgl. ebd., 201). Stausberg und Engler halten es sogar für eine wichtige Aufgabe der Religionswissenschaft, auf die Historizität des instrumentalen Denkens in Kategorien von Kosten und Nutzen hinzuweisen: „It is important to distinguish the strategic value of playing that game from the basic premise that no other game exists“ (ebd., 139). Diesem Ansatz folgend könnte auch das religionswissenschaftliche Studium nicht nur zur Ökonomisierung alternative Weltdeutungen behandeln, sondern sogar selbst eine darstellen und Lernziele ihres Studiums definieren, die weder unmittelbar berufsbezogen noch fachbezogen sind.

\subsection{Persönlichkeitsbildung als primärer Zweck der Harvard General Education}

Um die reale Möglichkeit der Umsetzung dieses Verständnisses des religionswissenschaftlichen Studiums aufzuzeigen, möchte ich den Leser in einen kleinen Exkurs in die amerikanische Universitätslandschaft entführen und mit den Lernzielen des General Education-Programms der Harvard University in Cambridge, USA, vertraut machen. Obwohl die Harvard University die zukünftige nicht nur intellektuelle, sondern vor allem auch ökonomische und politische Elite der USA ausbildet, betont sie Lernziele ihres Studiums jenseits der unmittelbaren Employability. So basiert die General Education im Bachelor-Studium auf der Idee einer grundlegenden liberal education, die einen engen Bezug zum realen Leben außerhalb der Universität haben soll (vgl. Harvard University Faculty of Arts and Sciences 2007). Ein wichtiges Lernziel ist es, mündige Bürger zu erziehen, die ein tiefgreifendes Verständnis der eigenen kulturellen Tradition und Geschichte besitzen, sich Informationen beschaffen und sie kritisch einordnen können, konstruktiv auf Veränderungen reagieren und Probleme lösen können sowie ethisch verantwortungsbewusst handeln und die Gesellschaft mitgestalten. Darüber hinaus verstehen die Mitglieder des Komitees, das sich jüngst mit den Zielen der 
General Education auseinandergesetzt hat, die Persönlichkeitsbildung als eine wesentliche Errungenschaft unserer Zivilisation. Sie wünschen sich Graduierte mit einem Bewusstsein für die Komplexität der Welt um sie herum und einem weiten Horizont, fähig zu kritischem und analytischem Denken, zu Reflexion und Selbstkritik sowie zur Kritik von Meinungen, Entscheidungen und Motivationen und mit der Bereitschaft, Bekanntes zu hinterfragen (Harvard University Faculty of Arts and Sciences 2007, 1-2.4-5). Damit setzt sogar die Elite-Universität eines Landes, das gemeinhin am stärksten mit Neoliberalismus und Kapitalismus assoziiert wird, auf idealistische Bildungswerte. Daran kann sich Europa ein Beispiel nehmen.

Allerdings kann das Konzept der General Education nicht eins zu eins auf deutsche Universitäten übertragen werden, da die Ausbildungsstrukturen in den USA sich fundamental vom deutschen und vielen europäischen Modellen unterscheiden. So ist ein normales undergraduate-Studium in den USA viel weniger fachbezogen als unsere Studiengänge. Die Studenten schreiben sich i.d. R. nicht für ein oder mehrere Fächer an der Universität ein, sondern für eine Studienrichtung wie die Liberal Education (die eher mit einem Studium Generale zu vergleichen ist). Gegen Ende dieses vierjährigen Studiums bilden sie einen oder zwei fachbezogene Schwerpunkte aus (den sogenannten „major“, in Harvard „concentration“ genannt). Erst die graduate studies (meist Master-Studiengänge) beziehen sich direkt auf ein Fach. In der Harvard General Education müssen die Studenten Kurse aus acht verschiedenen Disziplinbereichen belegen, die sowohl naturwissenschaftliche Disziplinen und die sogenannten Lebenswissenschaften als auch social studies und humanities einbeziehen. ${ }^{18}$ Der Vergleich ist simplifizierend, und vermutlich tue ich der Ausbildung an den High Schools unrecht - aber die General Education erinnert in vielem eher an das deutsche Abitur denn an unsere fachbezogenen Bachelor-Studiengänge. Neben der inhaltlichen Gewichtung ist auch die Pädagogik der General Education, zumindest in Harvard (vgl. Harvard University Faculty of Arts and Sciences 2007, 7.9.23), stärker an der Aktivität der Studenten und sogenannten hands-on Übungen orientiert, als es im Rahmen des deutschen, hoch-intellektuellen Konzepts von universitärem Lernen, gerade in den Geisteswissenschaften, denkbar wäre. ${ }^{19}$

18 Die Bereiche sind: „Aesthetic and Interpretive Understanding“, „Culture and Belief“, „Empirical Reasoning“, „Ethical Reasoning“, „Science of Living Systems“, „Science of the Physical Universe“, „Societies of the World“ und „The United States in the World“ (Harvard University Faculty of Arts and Sciences (2007, 7).

19 Für eine Diskussion der Frage, ob die General Education ihre selbstgesteckten Lernziele erreicht, aus Sicht eines Harvard-Absolventen vgl. Douthat (2005, 131-140). 


\subsection{Allgemeine Lernziele des religionswissenschaftlichen Studiums}

Aufbauend auf diesen allgemeinen Überlegungen zum Zweck eines universitären Studiums der Religionswissenschaft stelle ich nun einen ersten Vorschlag für Lernziele dieses Faches vor. In Ergänzung $\mathrm{zu}$ fachbezogenen Lernzielen und Schlüsselqualifikationen benenne ich hier grundlegende allgemeine Kompetenzen, die sowohl in den späteren Berufen der Graduierten relevant sein können als auch ihre Erziehung zu mündigen, verantwortungsbewussten Mitgliedern der Gesellschaft fördern und darüber hinaus ihre Persönlichkeit bilden. ${ }^{20}$ Zunächst werden Lernziele behandelt, welche die Religionswissenschaft mit anderen Geistes- und Kulturwissenschaften teilt; anschließend Lernziele, die charakteristisch für die Religionswissenschaft nach ihrem gegenwärtigen, kulturwissenschaftlichen Verständnis sind. ${ }^{21}$ Die Lernziele eines Studiengangs sind notwendigerweise recht abstrakt zu formulieren (vgl. Biggs und Tang 2009, 69-70) und grundsätzlich auf einer hohen Komplexitätsstufe angesiedelt.

\subsubsection{Allgemeine Lernziele der Kultur- und Geisteswissenschaften}

\section{Lernstrategien}

- Graduierte sollen verschiedene Lernstrategien und -techniken kennen, je nach Anforderungssituation flexibel auswählen und gezielt anwenden können. Darüber hinaus sollen sie ihre persönlichen Bedürfnisse in Bezug auf eine produktive Lernumgebung reflektieren können und fähig sein, ihre Motivation zu beeinflussen.

Für das Erreichen dieses Lernziels sind verschiedene Zwischenstufen notwendig: Zum einen sollte Studenten bewusst werden, dass wir in einer Wissensgesellschaft leben, in der sich nicht nur Wissensinhalte, sondern auch Methoden zur Wissenserlangung und Techniken der Wissensvermittlung schnell verändern und weiterentwickeln. Sich in dieser Umgebung zurechtzufinden, erfordert eine

20 Bauder-Begerow und Schäfer (2011b: 11) schließen unter Schlüsselkompetenzen nicht nur umfassendes Wissen, sondern auch langfristig erhaltene, übertragbare kognitive und praktische Fähigkeiten und individuelle Haltungen ein. Daran wird deutlich, dass die Grenzen zwischen allgemeinen Kompetenzen und Schlüsselkompetenzen fließend sind. Vgl. auch Chur (2011, 5455.58-62).

21 Da es hier um allgemeine Lernziele geht, ist natürlich nicht ausgeschlossen, dass diese nicht ebenfalls Lernziele anderer Disziplinen sein können. 
hohe reflexive Lernkompetenz und die Fähigkeit, sich lebenslang für Lernprozesse motivieren und begeistern zu können. Dieses Lernziel Lernen ist deshalb kein rein kognitives, sondern auch ein affektives Lernziel. Bisher wurde meist davon ausgegangen, dass Studenten diese Kompetenz schon mitbringen, oder ihr Erwerb wurde den Studenten selbst überlassen. Stattdessen ist es jedoch notwendig, Lernstrategien im Studium bewusst $\mathrm{zu}$ thematisieren und $\mathrm{zu}$ fördern.

\section{Informationen beschaffen und auswerten}

- Graduierte sollen fähig sein, eigenständig eine Frage- oder Problemstellung zu entwickeln und Strategien der Beschaffung von Informationen über verschiedene Sachverhalte kennen und einsetzen können. Dabei sollen sie Quellen kritisch kontextualisieren und einschätzen können. Die aus verschiedenen Texten gewonnenen Informationen sollen miteinander in Verbindung gebracht und die Ergebnisse in verständlicher Form anderen kommuniziert werden.

Dies ist sicherlich eine der klassischen Kompetenzen eines geisteswissenschaftlichen Studiums, das meist durch die Erstellung von Hausarbeiten eingeübt und bewertet wird. Während die DVRW zu Recht darauf hinweist, dass Graduierte heutzutage fähig sein müssen, Informationen schnell zu gewinnen (Deutsche Vereinigung für Religionswissenschaft 2011, 5), ist es m.E. ebenso wichtig, im Universitätsstudium auf die Gefahren der schnellen Informationsgewinnung hinzuweisen und die kritisch-kontextualisierende Sorgfalt im Umgang mit Quellen $\mathrm{zu}$ verinnerlichen. Davon unabhängig sollte eingeübt werden, die für wissenschaftliche Perspektiven typische Komplexität in eine allgemeinverständliche Sprache zu übersetzen.

\section{Die Besonderheit wissenschaftlichen Arbeitens und Denkens}

- Graduierte sollen erklären können, wodurch sich wissenschaftliches Denken und Arbeiten im Vergleich mit anderen Formen des Denkens und Argumentierens auszeichnet. Sie sollen wissenschaftliche Methoden gemäß einer Problemstellung auswählen und auf diese anwenden können. Des Weiteren sollen sie unterschiedliche Methoden, Empirie und Theorie miteinander zu verbinden, analysieren und wissenschaftliche Ansätze kontextualisieren und reflektieren können.

Wissenschaft ist eine Form des Denkens und Arbeitens, die sich von anderen unterscheidet: Sie ist eine Methode, um systematisch Wissen zu generieren; sie operiert anhand der Prinzipien Logik, Kohärenz und Rationalität und folgt be- 
stimmten Regeln (vgl. Thurfjell 2011, 212). ${ }^{22}$ Wissenschaft entwickelt (primär) sprachliche und visuelle Modelle von Wirklichkeit und vermittelt zwischen Daten, Fakten, Empirie auf der einen Seite und abstrakten Generalisierungen und Theorie(n) auf der anderen Seite. Während bisher das Wissen um diese Charakteristika meist nur implizit gelehrt und nur formale Grundtechniken wie Zitierweisen in Tutorien gezielt eingeübt werden, ist es ratsam, die Grundlagen und Prinzipien wissenschaftlichen Denkens explizit zu thematisieren, zusammen mit den Studenten historisch und kulturell zu kontextualisieren und zu reflektieren. Dadurch können Studenten eine deutlich höhere Verknüpfung zu anderen Lebens- und Kulturbereichen herstellen, und der kreative Umgang mit wissenschaftlichen Ansätzen außerhalb der Wissenschaft im engeren Sinne wird gefördert. Zudem ist es typisch für postmoderne und postkoloniale Epistemologien, Wissenschaft als nur eine Methode der Erkenntnisgewinnung unter anderen zu verstehen, deren Ergebnisse nicht prinzipiell universal gültig, sondern immer historisch und kulturell gebunden sind.

\section{Kritisches Denken und Problemlösen}

- Graduierte sollen kritisch denken, ihre Gedanken schriftlich und mündlich artikulieren und logisch und rational argumentieren können. Sie sollen fähig sein, imaginativ und kreativ intellektuelle Probleme zu lösen.

Diese Kompetenzen sind die Grundlage jedes wissenschaftlichen Arbeitens und zugleich fundamental für diverse Aufgaben in unserer Gesellschaft. Allerdings ist es unklar und umstritten, was genau mit kritischem Denken und Rationalität gemeint ist (vgl. Riveros et al. 2012; Toplak, West und Stanovich 2012), wodurch die Umsetzung dieser Kompetenz in Lehrstrategien und Prüfungsleistungen erschwert wird. Imaginatives und kreatives Denken wird zudem in den Wissenschaften nicht so hoch geschätzt wie z.B. in verschiedenen Kunstformen. Dennoch ist für intellektuelles Problemlösen nicht nur Methoden- und Sachkenntnis auf einer hohen Stufe der kognitiven Taxonomien erforderlich, sondern auch Imagination und Kreativität. Wie diese im Universitätsstudium gezielt gefördert werden können, muss weiter reflektiert werden (vgl. Ramsden 2003, 21-24.29).

\section{Komplexe Modelle von Wirklichkeit}

- Graduierte sollen ein Bewusstsein dafür entwickelt haben, dass Wissenschaft keine einfachen Antworten gibt, sondern im Vergleich zum Alltagsdiskurs

22 Die hier erfolgte Charakterisierung bildet natürlich nur einzelne Aspekte von Wissenschaft ab und ist von einem bestimmten Wissenschaftsverständnis geprägt. 
eine Komplexitätssteigerung erreicht. Sie sollen verschiedene Wahrheitsansprüche unterscheiden und Vermittlungs- und Legitimationsstrategien von Ideologien und Weltbildern analysieren können.

Wissenschaft im Sinne eines gegenwärtigen kulturwissenschaftlichen Weltbildes sucht zwar nach wie vor nach Regeln, Grundsätzen und großen Entwicklungslinien, versucht aber auch Mikroperspektiven einzunehmen sowie kulturelle und historische Besonderheiten differenziert einzufangen. Statt zu einfachen Antworten führt wissenschaftliche Forschung meist $\mathrm{zu}$ einer immensen Komplexitätssteigerung. Es ist wichtig, dass sich Studenten diese Einsicht erarbeiten und Hilfestellungen bekommen, die damit oft verbundenen Frustrationen zu bewältigen. Darüber hinaus scheint es gerade in unserer heutigen Welt zentral, dass Studenten den Einsatz von Stereotypen und andere narrative und legitimierende Strategien der Argumentation und des Wahrheitsanspruchs in Religion, Kultur und Wissenschaft erkennen, analysieren und reflektieren können.

\subsubsection{Disziplinbezogene allgemeine Lernziele}

\section{Religionswissenschaftliches Denken und Arbeiten}

- Graduierte sollen ein Bewusstsein für religionswissenschaftliches Denken und Argumentieren entwickelt haben. Dieses Bewusstsein soll sie befähigen, eine Fragestellung aus religionswissenschaftlicher Perspektive und in Bezug auf gegenwärtig in der Disziplin diskutierte Debatten zu entwickeln. Sie sollen wissenschaftliche Methoden auswählen und anwenden können, die zur Beantwortung dieser Frage beitragen.

Bezüglich dieses Lernziels ist es notwendig anzumerken, dass es in der Religionswissenschaft eine umfangreiche Diskussion darüber gibt, ob Religionswissenschaft über eine spezifische, eigene Methode verfügt und ob sie überhaupt eine eigenständige Disziplin ist oder eher als Forschungsfeld zu bezeichnen ist (vgl. im Zusammenhang mit der Ausrichtung von Studiengängen Berner 2011, 153-156; Engler und Stausberg 2011, 129). Insofern kann es weniger Lernziel sein, dass Studenten „wie ein Religionswissenschaftler“ denken lernen (wenn wir gar nicht wissen, was das sein soll, kann, darf etc.) und sich von anderen Disziplinen abgrenzen können. Stattdessen sollen sie für dieses Grundsatzproblem sensibilisiert worden sein und Positionen innerhalb der Debatte erklären, kontrastieren, bewerten, reflektieren und vertreten können. 


\section{Interdisziplinarität und Vielfalt der Perspektiven}

- Graduierte sollen mit den Sprachen, Denkweisen und Methoden verschiedener wissenschaftlicher Perspektiven vertraut sein, sie in eigenen Worten erklären und ihre Charakteristika voneinander abgrenzen können. Sie sollen einen eigenen religionsgeschichtlichen und methodischen Schwerpunkt gewählt und soweit ausgearbeitet haben, dass sie innerhalb dessen wissenschaftlich argumentieren können. Gleichzeitig sollen sie fähig sein, sich mit Vertretern anderer Disziplinen über einen Sachverhalt zu verständigen und sich flexibel in der Vielfalt der Perspektiven bewegen.

Die Fähigkeit zu interdisziplinärem Denken wird in den letzten Jahren als Kernkompetenz fast jedes Universitätsstudiums eingefordert. Über die Lehrstrategien zur Förderung dieser Kompetenz wird jedoch seltener reflektiert. In der religionswissenschaftlichen Debatte um ihre eigene Identität wird oft gerade die Vielfalt der Perspektiven als besonderes Charakteristikum des Faches ausgemacht (wenn sie schon Schwierigkeiten hat, ihren Gegenstand zu definieren und keine eigene Methode aufweist). Die Vielfalt der Perspektiven ist u.a. auch dem Umstand geschuldet, dass die Religionsgeschichte in Vergangenheit und Gegenwart ein riesiges Feld mit einer Vielzahl von Aspekten ist. Wenn wir diese Besonderheit der Religionswissenschaft bewusst in Lehrstrategien umsetzen, können wir die Fähigkeit zur intellektuellen Flexibilität bei unseren Studenten fördern und zu ihrem Markenzeichen gegenüber Graduierten anderer Fächer werden lassen.

\section{Unterschiedliche Wissens- und Ausdrucksformen}

- Graduierte sollen verschiedene Genres von Wissensformen und -artikulationen erkennen und Methoden ihrer Analyse auswählen können. Darüber hinaus sollen sie die in Religionen verwendeten Ausdrucksformen und Inhalte durch Analyse ihres Zusammenhangs so verstehen können, dass sie diese in Kommunikationsmuster übersetzen können, auf die auch nicht-Angehörige der jeweiligen Religion zurückgreifen.

Für die Entwicklung einer kulturwissenschaftlichen Religionswissenschaft war ihre Abgrenzung von theologischen, krypto-theologischen und religiösen Ansätzen ein wesentlicher Schritt ihrer Identitätsbildung. Deshalb gehört es zu den wichtigen Lernzielen in einem religionswissenschaftlichen Studium, verschiedene Wissensformen, Erkenntnisinteressen, Epistemologien und Darstellungsformen von Wissen in Texten analysieren und reflektieren zu können. Darüber hinaus wies Bochinger darauf hin, dass manche religiösen Aussagen nur innerhalb der jeweiligen Tradition verständlich sind, weil sie auf „sprachliche und andere Codes“ zurückgreifen, die „nicht zum allgemeinen Kommunikationsmus- 
ter einer modernen Gesellschaft gehören“ (Bochinger 16./17. Juni 2010, 4). Religionswissenschaftler sollen fähig sein, zwischen diesen sprachlichen und nonverbalen Kommunikationsformen übersetzen und vermitteln zu können.

\section{Regeln religionswissenschaftlichen Argumentierens}

- Graduierte sollen Regeln akademischen Diskutierens, die spezifisch für die kulturwissenschaftliche Religionswissenschaft sind, mündlich und schriftlich anwenden können.

David Thurfjell hat einen faszinierenden Artikel über die Herausforderung religionswissenschaftlicher Hochschuldidaktik mit multikultureller Studentenschaft geschrieben (Thurfjell 2011). Seiner Erfahrung nach setzen religionswissenschaftliche Dozenten in vielen Fällen unbewusst eine vorherige Prägung der Studenten durch eine ,säkulare post-judäo-christliche Weltsicht' voraus (ebd., 209). Dadurch fühlen sich Studenten, die von anderen kulturellen und religiösen Werten geprägt wurden, oft ausgeschlossen. Deshalb haben sich die Dozenten an seinem Department for the Study of Religions der Södertörn University (Schweden) zur Aufgabe gemacht, eine Atmosphäre des gegenseitigen Respekts und gemeinsamen Lernens aufzubauen. Sie betonen, dass jeder Student gleich seiner Herkunft bestimmte Regeln akademischen Denkens, Reflektierens, Artikulierens und Argumentierens lernen und einüben muss. Zu diesen Regeln zählen neben der Quellenkritik auch die Selbstreflexion, das Einnehmen einer nicht-konfessionellen und nichtnormativen Perspektive im Unterrichtsraum und der „as-if-approach“: ein intellektuelles Spiel, in dem man sich in die Weltsicht von Religionen sowie von religionswissenschaftlichen Theorien hineinversetzt und lernt auf der Grundlage ihrer Prämissen zu denken und zu argumentieren. Durch dieses Vorgehen wollen die Dozenten vermitteln, dass im Unterricht alle Perspektiven auf die Welt gleich behandelt werden (ebd., 212-214).

\section{Kulturelle und religiöse Kompetenz}

- Graduierte sollen eine hohe kulturelle Kompetenz insbesondere in Bezug auf Religionen aufweisen. Dazu gehört:

- die Bereitschaft und Fähigkeit zur Reflexion über die eigene und „fremde“ Kulturen und Religionen;

- Religionen als Welterklärungsmodelle wahrzunehmen, die für viele Menschen eine wichtige Rolle in ihrem Leben spielen. Dafür sollen Graduierte verschiedene Beispiele kennengelernt haben sowie erklären und miteinander vergleichen können. Die dabei gewonnenen Einsichten sollen sie auf weitere Fallbeispiele und Situationen im Alltag und in der Weltpolitik übertragen können; 
- Religionen als wichtige Handlungsmotivation und Faktor in menschlichem Verhalten in der Geschichte und in der globalisierten, pluralisierten Gegenwart wahrzunehmen. Dafür sollen Graduierte verschiedene Beispiele durchdrungen haben und die dabei gewonnenen Erkenntnisse übertragen können;

- Zusammenhänge zwischen Kultur, Religion, Politik, Kunst und anderen gesellschaftlichen Teilbereichen in ihren historischen Zusammenhängen verstehen, analysieren und bewerten zu können und die historische und kulturelle Bedingtheit des Modells der Trennung gesellschaftlicher Teilbereiche reflektieren zu können;

- Narrative über religiöse und kulturelle Identitäten verstehen, erklären und analysieren zu können sowie auf dessen Grundlage Stereotypen auflösen zu können.

\section{Abschluss}

Der vorliegende Artikel verfolgte die Absicht, in der Religionswissenschaft eine Diskussion über die Bedeutung der Hochschuldidaktik für unsere Lehre und deren gesellschaftliche Relevanz anzuregen, indem grundlegende didaktische Erkenntnisse beispielhaft umgesetzt wurden. Auf der Grundlage des Ansatzes des Deep Learning wurde das Modell des Constructive Alignment von Lernzielen, Lehrstrategien und Prüfungen vorgestellt und der Blickwinkel auf den Teilbereich von Lernzielen fokussiert. Nach einer Einführung in Regeln zur Formulierung von Lernzielen wurden einzelne allgemeine Lernziele für das religionswissenschaftliche Studium entworfen.

Durch diese pragmatische Umsetzung der Erkenntnisse einiger hochschuldidaktischer Klassiker in Bezug auf die Frage von Lernzielen möchte ich die Diskussion über angemessene Didaktik in unserem Fach anstoßen. Didaktik betrifft alle Universitätsdozenten, nicht nur diejenigen, deren Forschungsschwerpunkt der Religionsunterricht ist.

Als Fazit des hier vorgestellten, ersten Entwurfs von Lernzielen des religionswissenschaftlichen Studiums lässt sich festhalten, dass diese weite Perspektive auf den Sinn und Zweck dieses Studiums und seiner Lernziele geradezu zwangsläufig zu der Erkenntnis führt, dass nur die Ergebnisse eines Deep Learning von den Studenten nach Abschluss des Studiums zu gebrauchen sind. Egal, ob es sich um allgemeine Lernziele, Schlüsselkompetenzen oder fachbezogene Inhalte handelt - wir brauchen Studienabgänger, die eigenständig mit Lehrinhalten umgehen und sie nicht nur anwenden, sondern sie auch reflektieren, in Kontext setzen und ihre Prinzipien auf andere Wissensbereiche und Tätigkeiten übertra- 
gen können. Dies allerdings ist nur möglich, wenn ein hoher Grad ihrer Aneignung erreicht ist und die Aneignung im Sinne des tiefenorientierten Lernens in die Tiefe geht.

Einen sogenannten disclaimer zum Abschluss: Dieser Artikel spiegelt sowohl meine Eindrücke wider, die ich in Gesprächen mit Kollegen und Studenten verschiedener religionswissenschaftlicher Standorte gewonnen habe, als auch die Ergebnisse einer Recherche bisher erschienener Publikationen. Möglicherweise finden an einzelnen religionswissenschaftlichen Instituten schon viel mehr didaktische Diskussionen, Reflexionen und Umsetzungen statt, die ich hier nicht berücksichtigt habe. Deshalb möchte ich dazu anregen, sich über diese Bemühungen im Fachkollegium auszutauschen. Des Weiteren erhebt mein Entwurf allgemeiner Lernziele des religionswissenschaftlichen Studiums weder den Anspruch, vollständig zu sein, noch jede epistemologische oder andere Diskussion in Teilbereichen der Religionswissenschaft berücksichtigt zu haben. Er ist notwendigerweise simplifizierend. Sehr hilfreich wäre es z. B. in einer zukünftigen Arbeit die Menschenbilder $\mathrm{zu}$ reflektieren und historisch $\mathrm{zu}$ kontextualisieren, die den derzeit anerkannten hochschuldidaktischen Theorien und unserem Verständnis von universitärer Bildung zugrunde liegen. In diesem Zusammenhang stellen sich auch die grundlegenden Fragen nach der Identität der Religionswissenschaft und unserem allgemeinen Wissenschaftsverständnis.

Mein Anliegen war es hier, einen ersten Schritt in Richtung einer pragmatischen Umsetzung unserer komplexen Fachinhalte in die Formulierung von Lernzielen vorzunehmen. Wenn der Artikel es vermag, vielfältige Widersprüche auszulösen und eine lebhafte Diskussion über unsere Fachdidaktik anzuregen, hat er seinen Zweck erfüllt.

\section{Literaturverzeichnis}

Alberts, Wanda. 2008a. „Didactics of the Study of Religions.“ Numen 55: 300-334.

Alberts, Wanda. 2008b. „Editorial.“ In Michael Stausberg, Hg., Sonderheft The Challenge of Religious Education for the History of Religions. Numen 55: 121-122.

Alles, Gregory D. 2011. „What (kind of) good is Religious Studies?“ Religion 41/2: 217-223.

Bauder-Begerow, Irina und Stefanie Schäfer, Hg. 2011a. Learning 9/11: Teaching for Key Competences in Literary and Cultural Studies. Heidelberg: Universitätsverlag Winter.

Bauder-Begerow, Irina und Stefanie Schäfer, Hg. 2011b. „Learning 9/11: Teaching for Key Competences in Literary and Cultural Studies.“ In Learning 9/11: Teaching for Key Competences in Literary and Cultural Studies, hg. v. Irina Bauder-Begerow und Stefanie Schäfer, 7-25. Heidelberg: Universitätsverlag Winter.

Bereiter, Carl und Marlene Scardamalia. 2012. „Theory Building and the Pursuit of Understanding in History, Social Studies, and Literature.“ In Enhancing the Quality of Learning: Dispositi- 
ons, Instruction, and Learning Processes, hg. v. John R. Kirby und Michael J. Lawson, 160-177. Cambridge/New York/Melbourne et al.: Cambridge University Press.

Berner, Ulrich. 2011. „Contextualization of Religious Studies and of religious phenomena.“ Religion 41/2: 149-157.

Biggs, John Burville. 2012 „Enhancing Learning through Constructive Alignment.“ In Enhancing the Quality of Learning: Dispositions, Instruction, and Learning Processes, hg. v. John R. Kirby und Michael J. Lawson, 117-136. Cambridge/New York/Melbourne et al.: Cambridge University Press.

Biggs, John Burville und Catherine Tang. ${ }^{3} 2009$. Teaching for quality learning at university: What the student does. Maidenhead: McGraw-Hill.

Bleisch, Petra und Dirk Johannsen. „Interessengeleitetes Studium: Eine deskriptorenbasierte Neugestaltung religionswissenschaftlicher Einführungsseminare.“ Verfügbar (12.06.2014) unter http://www.academia.edu/3142424/Interessengeleitetes_Studium_PBDJ.

Bloom, Benjamin Samuel. 1956-1964. Taxonomy of Educational Objectives: The Classification of Educational Goals. Vol. 1, Cognitive Domain. Vol. 2, Affective Domain. New York: McKay.

Bochinger, Christoph. 2010. „Vielfalt der Religionen und religionswissenschaftliche Kompetenz: Die Empfehlungen des Wissenschaftsrats im Horizont säkularer Kulturwissenschaften.“ Vortrag, Berlin, 16.06.2010. Verfügbar (12.06.2014) unter: www.wissenschaftsrat.de/ download/archiv/Bochinger.pdf.

Bundesministerium für Bildung und Forschung, Hg. Der Bologna-Prozess: eine europäische Erfolgsgeschichte. Verfügbar (12.06.2014) unter: http://www.bmbf.de/de/3336.php\#top.

Chur, Dietmar. 2011. „Developing Key Competences in Higher Education.“ In Learning 9/11: Teaching for Key Competences in Literary and Cultural Studies, hg. v. Irina Bauder-Begerow und Stefanie Schäfer, 53-74. Heidelberg: Universitätsverlag Winter.

Deutsche Vereinigung für Religionswissenschaft. 2011. „Empfehlungen für die Konzeption religionswissenschaftlicher Studiengänge.“ Verfügbar (12.06.2014) unter http://www.dvrw.unihannover.de/fileadmin/dvrw/pdf/300112_Rahmenordnung.pdf

Douthat, Ross Gregory. 2005. Priviledge: Harvard and the Education of the Ruling Class. New York: Hyperion.

Engler, Steven und Michael Stausberg. 2011. „Introductory Essay. Crisis and creativity: Opportunities and threats in the global study of religion/s." Religion 41/2: 127-143.

Frank, Katharina und Christoph Bochinger. 2008. „Religious Education in Switzerland as a Field of Work for the Study of Religions: Empirical Results and Theoretical Reflections.“ Numen 55: 183-217.

Führding, Steffen, Konrad Boidol, Dorothee Christiani, Svenja Sabrina Lehmann, Katharina Lemme, Sonja Matthies, Julia Schneider, Stefan Schröder und Martin Trappe. 2009. Warum Religionswissenschaft? Eine empirische Studie über die Gründe, Religionswissenschaft zu studieren. Marburg: Diagonal-Verlag.

Harvard University Faculty of Arts and Sciences. 2007. „Report of the Task Force on General Education.“ Cambridge, MA.

Kirby, John R. und Michael J. Lawson. Hg. 2012a. Enhancing the Quality of Learning: Dispositions, Instruction, and Learning Processes. Cambridge/New York/Melbourne et al.: Cambridge University Press.

Kirby, John R. und Michael J. Lawson. Hg. 2012b. „Introduction.“ In Enhancing the Quality of Learning: Dispositions, Instruction, and Learning Processes, hg. v. John R. Kirby und Michael J. Lawson, 1-14. Cambridge/New York/Melbourne et al.: Cambridge University Press. 
Lawson, Michael J. und Helen Askell-Williams. 2012. „Framing the Features of Good-Quality Knowledge for Teachers and Students." In Enhancing the Quality of Learning: Dispositions, Instruction, and Learning Processes, hg. v. John R. Kirby und Michael J. Lawson, 137-159. Cambridge/New York/Melbourne et al.: Cambridge University Press.

Macke, Gerd, Ulrike Hanke und Pauline Viehmann. 2008. Hochschuldidaktik: Lehren, vortragen, prüfen. Weinheim/Basel: Beltz Verlag.

Nünning, Vera, Hg. 2008. Schlüsselkompetenzen: Qualifikationen für Studium und Beruf. Stuttgart/Weimar: Metzler.

Ramsden, Paul. ${ }^{2} 2003$. Learning to Teach in Higher Education. London/New York: Routledge/ Falmer.

Riveros, Augusto, Stephen P. Norris, Denyse V. Hayward und Linda M. Phillips. 2012. „Dispositions and the Quality of Learning.“ In Enhancing the Quality of Learning: Dispositions, Instruction, and Learning Processes, hg. v. John R. Kirby und Michael J. Lawson, 32-50. Cambridge/New York/Melbourne et al.: Cambridge University Press.

Stausberg, Michael. 2011. „The Bologna process and the study of religion/s in (Western) Europe.“ Religion 41/2: 187-207.

Thurfjell, David. 2011. „Religionswissenschaft and the challenge of multi-religious student groups.“ Religion 41/2: 209-216.

Toplak, Maggie E., Richard F. West und Keith E. Stanovich. 2012. „Education for Rational Thought." In Enhancing the Quality of Learning: Dispositions, Instruction, and Learning Processes, hg. v. John R. Kirby und Michael J. Lawson, 51-92. Cambridge/New York/ Melbourne et al.: Cambridge University Press. 